\title{
miR-449c inhibits migration and invasion of gastric cancer cells by targeting PFKFB3
}

\author{
${\text { XIN } \text { CHEN }^{1}, \text { ANPING WANG }}^{2}$ and XINXIA YUE ${ }^{3}$ \\ ${ }^{1}$ Department of Digestive Disease, People's Hospital Xinjiang Autonomous Republic, Urumqi, Xinjiang 830001; \\ ${ }^{2}$ Department of Anesthesia, The Second Affiliated Hospital of Xinjiang Medical University, Urumqi, Xinjiang 830054; \\ ${ }^{3}$ Department of Digestive Disease, People's Hospital Xinjiang Autonomous Republic, \\ North Hospital, Urumqi, Xinjiang 830001, P.R. China
}

Received March 5, 2017; Accepted October 20, 2017

DOI: $10.3892 / \mathrm{ol} .2018 .8609$

\begin{abstract}
RNAs (miRNAs) are important regulators in the development and progression of human gastric cancer (GC). However, the expression and biological function of miR-449c in $\mathrm{GC}$ remains unknown. In the present study, miR-449c was demonstrated to be downregulated in GC tissues and cell lines compared with normal. Decreased miR-449c levels were associated with poor prognosis in GC patients. Overexpression of miR-449c inhibited migration and invasion in SGC-7901 cells, while inhibition of miR-449c enhanced migration and invasion in MGC-803 cells. Furthermore, 6-phosphofructo-2-kinase (PFKFB3) was identified to be a downstream target of miR-449c in GC cells. miR-449c directly regulated the expression of PFKFB3 in GC cells. Overexpression of PFKFB3 abrogated the inhibitory effect of miR-449c on the migration and invasion of GC cells. In conclusion, the present study suggests that miR-449c may be a novel biomarker and potential therapeutic target in GC.
\end{abstract}

\section{Introduction}

Gastric cancer (GC), the fourth most common type of cancer in humans, is the second leading cause of cancer-related deaths worldwide (1). The prognosis in GC patients is dismal, especially for those with advanced TNM stages (2). The main reason for the unsatisfactory prognosis in GC patients is the occurrence of local and systemic metastasis. However, the molecular mechanisms underlying GC metastasis remain largely unknown. Investigating the mechanisms and regulatory networks of GC metastasis will contribute to the identification of novel biomarkers and therapeutic targets of GC.

Correspondence to: Dr Anping Wang, Department of Anesthesia, The Second Affiliated Hospital of Xinjiang Medical University, 1284 Qidaowan Road, Urumqi, Xinjiang 830054, P.R. China

E-mail: 1dnin1998@163.com

Key words: miR-449c, gastric cancer, metastasis, 6-phosphofructo-2-kinase
MicroRNAs (miRNAs) are a group of short noncoding RNA sequences that can post-transcriptionally regulate the expression of target genes $(3,4)$. Previous studies have demonstrated that miRNAs are involved in regulating several cellular functions, including cell proliferation, apoptosis, motility and differentiation $(5,6)$. Abnormal expression and function of miRNAs has been confirmed to serve important roles in different types of human cancers, including GC $(7,8)$. Multiple miRNAs are regarded as attractive biomarkers and potential therapeutic targets for GC $(9,10)$.

miR-449c was recently reported to have important roles in human cancer, such as non-small cell lung cancer (11) and liver cancer (12). miR-449c was demonstrated to inhibit the proliferation and invasion ability of non-small cell lung cancer cells by targeting MYC proto-oncogene, bHLH transcription factor (c-Myc) (11). In liver cancer, miR-449c was demonstrated to target SYR-box 4 (SOX4) and to inhibit the epithelial-mesenchymal transition and metastasis of liver cancer cells (12). However, the expression and function of miR-449c in GC remains unknown.

In the present study, miR-449c was demonstrated to be downregulated in GC tissues and cell lines compared with normal. Decreased miR-449c levels were associated with poor prognosis in GC patients. Overexpression of miR-449c inhibited, while knockdown of miR-449c promoted, the migration and invasion of GC cells. Furthermore, the results demonstrated that 6-phosphofructo-2-kinase (PFKFB3) was a direct downstream target of miR-449c in GC cells, and that overexpression of PFKFB3 could abrogate the inhibitory effect of miR-449c mimic on the migration and invasion of GC cells.

\section{Materials and methods}

Clinical specimens and cell culture. GC tissues and adjacent non-tumor tissues were collected from GC patients who received surgical treatments between 2004 and 2012 in the Department of Digestive Disease, The Second Affiliated Hospital of Xinjiang Medical University (Xinjiang, China). The age range of the patients with GC was 24-79 years old (mean age, 36.8 years old), with a male/female ratio of 1.5 (males, 36; females, 24). None of the patients received chemotherapy prior to surgical treatment. The clinical samples were 
collected with informed consent from all patients. The specimens were stored in liquid nitrogen prior to being subjected to further experiments. The protocols for collecting and using the clinical specimens were approved by the Institutional Research Ethics Committee of the Second Affiliated Hospital of Xinjiang Medical University (Urumqi, China).

The normal gastric epithelial GES-1 cell line and four GC cell lines (AGS, SGC-7901, MKN-45 and MGC-803) were purchased from American Type Culture Collection (Manassas, VA, USA) and the Cell Bank of Type Culture Collection of the Chinese Academy of Sciences (Shanghai, China). The cells were cultured in RPMI1640 medium (Gibco; Thermo Fisher Scientific, Inc., Waltham, MA, USA) supplemented with $10 \%$ fetal bovine serum (Gibco; Thermo Fisher Scientific, Inc.), $100 \mathrm{mg} / \mathrm{ml}$ penicillin and $100 \mathrm{mg} / \mathrm{ml}$ streptomycin. All cell cultures were maintained in a humidified atmosphere with $5 \%$ $\mathrm{CO}_{2}$ at $37^{\circ} \mathrm{C}$.

Cell transfection. miR-449c mimic (cat no. miR10013771-1-5), miR-449c inhibitor (cat no. miR20010251-1-5) and corresponding control vectors (cat nos. miR01201-1-5 and miR02201-1-5) were purchased from Guangzhou RiboBio Co., Ltd (Guangzhou, China). For miRNA transfection, the final concentration transfected was $50 \mathrm{nM}$. pcDNA3.1-PFKFB3 and empty control vector were bought from GeneChem Co. Ltd. (Shanghai, China). For plasmid transfection, the final concentration transfected was $1.3 \mu \mathrm{g} / \mathrm{ml}$. Transfection into GC cells was performed with lipofectamine 2000 (Invitrogen; Thermo Fisher Scientific, Inc.) according to the manufacturer's protocol. Following $48 \mathrm{~h}$ after transfection, the cells were used for further experiments.

Reverse transcription-quantitative polymerase chain reaction $(R T-q P C R)$. Total RNA from clinical tissues was extracted using an RNA isolation kit (cat. no. AP-MN-MS-RNA; Corning Inc., Corning, NY, USA). Total RNA and miRNAs from GC cells were extracted using TRIzol Reagent (Thermo Fisher Scientific, Inc.) and microRNA purification kit (Norgen Biotek Corp., Thorold, ON, Canada) respectively, according to the manufacturer's protocol. cDNA reverse transcription was performed with High-Capacity cDNA Reverse Transcription kit (Applied Biosystems; Thermo Fisher Scientific, Inc.). qPCR for miR-449c was performed using Taqman microRNA assay primers (Applied Biosystems; Thermo Fisher Scientific, Inc.), while qPCR for PFKFB6 mRNA was performed using SYBR Green PCR Master Mix (Applied Biosystems; Thermo Fisher Scientific, Inc.). U6 and GAPDH were used as internal controls for miR-449c and PFKFB6, respectively. The thermocycling conditions were as follows: $95^{\circ} \mathrm{C}$ for $30 \mathrm{sec}$, followed by 40 cycles at $95^{\circ} \mathrm{C}$ for $5 \mathrm{sec}$, then $60^{\circ} \mathrm{C}$ for $30 \mathrm{sec}$. Relative fold changes in mRNA expression were calculated using the $2^{-\triangle \Delta C q}$ method (13). Primers for miR-449c, PFKFB3, U6 and GAPDH were purchased from GenePharma Co., Ltd. (Shanghai, China). The primer sequences were as follows: MiR-449c forward, 5'-CGCGGATCCTAATGCAATCGT TTGCATCTG-3' and reverse, 5'-CCGGAATTCTGGGTT TGGTCTTTCAAGGAG-3'; U6 forward, 5'-TGCGGGTGC TCGCTTCGGCAGC-3' and reverse, 5'-CAGTGCAGG GTCCGAGGT-3'; PFKFB3 forward, 5'-CCTCACTCGCAG CCACTTCT-3' and reverse, 5'-CAGTTCCTACTCAATTCC
AA-3'; GAPDH forward, 5'-TGGGTGTGAACCACGAGA A-3' and reverse, 5'-GGCATGGACTGTGGTCATGA-3'.

Protein extraction and western blot analysis. Total cellular protein was extracted using radioimmunoprecipitation assay lysis buffer containing $50 \mathrm{mmol} / \mathrm{l}$ Tris- $\mathrm{Cl}(\mathrm{pH} 7.5), 0.2 \mathrm{mmol} / \mathrm{l}$ EDTA, $150 \mathrm{mmol} / \mathrm{l} \mathrm{NaCl}, 1 \mathrm{mmol} / \mathrm{l} \mathrm{PMSF}$ and $1 \%$ Nonidet-P40 supplemented with protease inhibitor. The bicinchoninic acid kit (Pierce; Thermo Fisher Scientific, Inc.) was used for quantification of protein concentration. A total of $30 \mu \mathrm{g}$ cellular proteins were loaded on 4-20\% SDS-PAGE for protein separation. The separated protein samples were transferred to polyvinylidene fluoride membranes. Then, the membranes were blocked with $5 \%$ non-fat milk for $1 \mathrm{~h}$ at room temperature, and then incubated with the following antibodies overnight at $4^{\circ} \mathrm{C}$ : PFKFB3 (1:1,000; cat no. \#9645; Cell Signaling Technologies, Inc., Danvers, MA, USA) and GAPDH (1:2,000; cat no. \#5174; Cell Signaling Technologies, Inc.). The membranes were then incubated with secondary mouse anti-rabbit (cat. no., 3678) or goat anti-mouse (cat. no., 58802) antibodies $(1: 2,000)$ conjugated with horseradish peroxidase (Cell Signaling Technologies, Inc.) at room temperature for $1 \mathrm{~h}$. The signal intensity of protein bands was visualized with enhanced chemiluminescence reagents (GE Healthcare, Chicago, IL, USA). Image J software (version 1.41; National Institutes of Health, Bethesda, MD, USA) was used to quantify signal intensity.

Migration and invasion assays. Boyden chambers/transwell assays were used to measure the migratory ability of GC cells, following the manufacturer's protocol. Matrigel-coated transwell inserts with $8 \mu \mathrm{m}$ pores (Nalge Nunc International, Penfield, NY, USA) were used for measuring the invasive ability of GC cells. The same $8 \mu \mathrm{m}$ pore inserts were used for migration as well, with the addition of Matrigel coating. Transwell inserts were coated with $100 \mu$ l Matrigel (1:8 dilution in RPMI1640 medium). GC cells were suspended in serum-free RPMI-1640 medium ( $10 \times 10^{4}$ cells per $500 \mu \mathrm{l}$ serum-free media) and were seeded in the upper chamber of the transwell inserts. A total of $800 \mu$ l RPMI1640 containing $10 \%$ serum was added in the lower chamber. Following $24-48 \mathrm{~h}$ of incubation, crystal violet staining (5 min at room temperature) was used for the identification of the invaded GC cells. A Leica light microscope was used to count the migrated or invaded cells at the magnification of x200. A total of nine optical fields were counted per sample, with at least three samples per group.

Luciferase reporter assay. Wild type (wt) or mutant (mt) PFKFB3 3'-UTR was cloned into the pmiR-RB-REPORT ${ }^{\text {TM }}$ luciferase vector (Promega Corporation, Madison, WI, USA). The luciferase reporting vectors containing the interacting sequences of PFKFB3 were co-transfected with miR-449c mimic or miR-449c inhibitor into GC cells in 6-well plates using Lipofectamine 2000 (Invitrogen; Thermo Fisher Scientific, Inc.). At $48 \mathrm{~h}$ post-transfection, the luciferase activity was measured using the dual-luciferase reporter assay system (Promega Corporation). Renilla luciferase activity was normalized to firefly luciferase activity.

Bioinformatics analysis. Targetscan database (http://www.targetscan.org/vert_71/) was searched to 
A

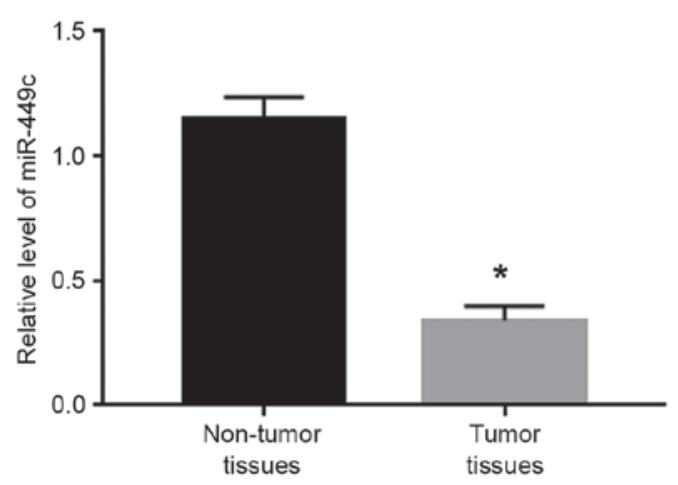

C

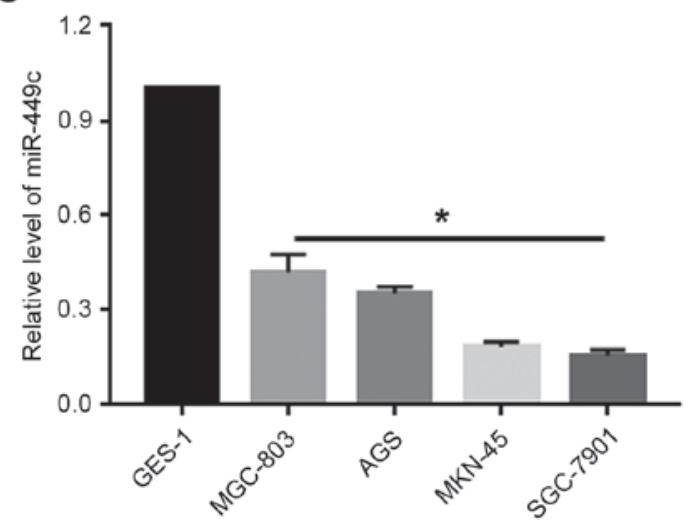

B

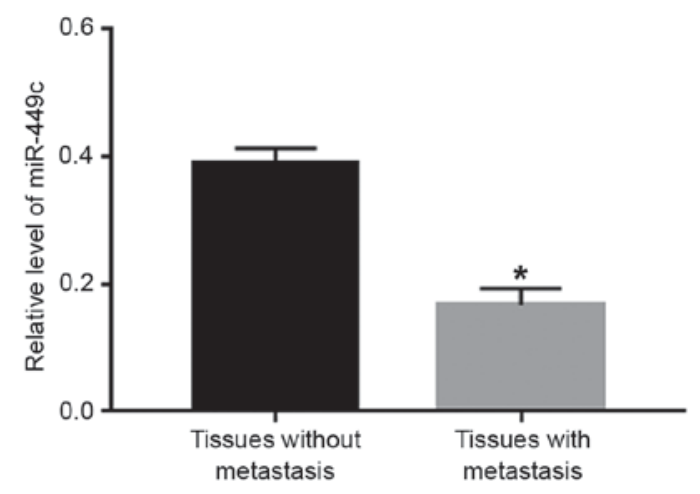

D

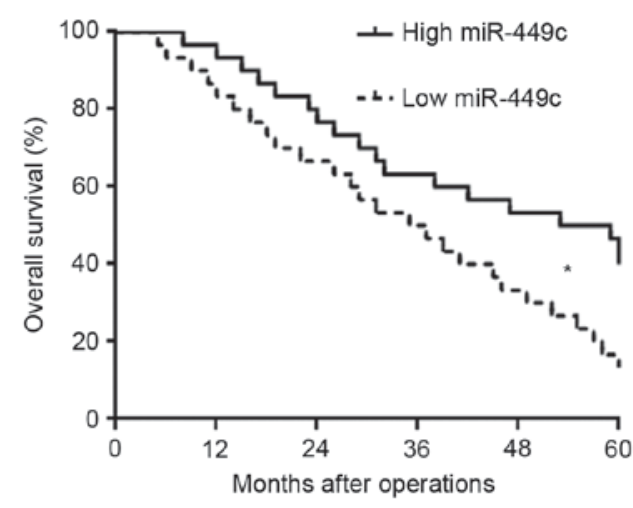

Figure 1. miR-449c is downregulated in GC tissues and cells. (A) The relative expression of miR-449c in 60 paired primary GC tissues and adjacent non-tumor tissues was compared. ${ }^{*} \mathrm{P}<0.05$. (B) The relative expression of miR-449c in tumor tissues with metastasis was compared to tumor tissues without metastasis. ${ }^{*} \mathrm{P}<0.05$. (C) The relative expression of miR-449c in GC cell lines was compared to the normal gastric epithelial cells GES-1. * $<<0.05$. (D) Kaplan-Meier analysis of miR-449c expression levels in GC patients. ${ }^{*} \mathrm{P}<0.05$. GC, gastric cancer.

identify the potential downstream target of miR-449c on November 2016. The search term used was 'miR-449c'. The complimentary sequences mediating the binding between miR-449c and PFKFB3 were identified using the Targetscan database.

Statistical analysis. All statistical analyses in the present study were performed using the GraphPad Prism 5 software (GraphPad Software, Inc., La Jolla, CA, USA). Quantitative data were presented as mean \pm standard error of the mean, from replicate experiments $(n>3)$. Kaplan-Meier analysis was performed to evaluate the prognostic significance of miR-449c in GC. Statistical significance was analyzed using the Chi-square and Student's t-test. $\mathrm{P}<0.05$ was considered to indicate a statistically significant difference.

\section{Results}

miR-449c expression is downregulated in GC. To investigate the expression levels of miR-449c in GC, qPCR was used to measure miR-449c levels in GC tissues and adjacent non-tumor tissues. The results from the qPCR analysis demonstrated that, compared with adjacent non-tumor tissues, GC tissues exhibited significantly decreased levels of miR-449c $(\mathrm{P}<0.05$; Fig. 1A). Next, the GC tissues were subdivided into two groups: cases with metastasis and cases without metastasis. Compared with the non-metastatic cancer tissues, tissues from patients with metastasis exhibited significantly decreased levels of miR-449c ( $\mathrm{P}<0.05$; Fig. 1B). Furthermore, the expression levels of miR-449c were measured in several GC cell lines, including MGC-803, AGS, MKN-45 and SGC-7901. Compared with the normal gastric epithelial cell line GES-1, the miR-449c expression levels were significantly reduced $(\mathrm{P}<0.05$; Fig. $1 \mathrm{C})$. Of note, Kaplan-Meier analysis revealed that decreased miR-449c expression was significantly associated with decreased overall survival rate in $\mathrm{GC}$ patients $(\mathrm{P}<0.05$; Fig. 1D).

Overexpression of miR-449c inhibits migration and invasion in GC cells. As illustrated in Fig. 1C, the expression levels of miR-449c were the lowest in SGC-7901 cells. Therefore, SGC-7901 cells were transfected with miR-449c mimic. Overexpression of miR-449c mimic significantly increased the expression level of miR-449c in SGC-7901 cells $(\mathrm{P}<0.05$; Fig. 2A). Then, transwell assays were performed to evaluate the effect of miR-449c overexpression on the migration and invasion ability of the SGC-7901 cells. The results demonstrated that overexpression of miR-449c significantly decreased both the migration $(\mathrm{P}<0.05$; Fig. $2 \mathrm{~B})$ and the invasion of SGC-7901 cells $(\mathrm{P}<0.05$, Fig. $2 \mathrm{C})$, compared with cells transfected with control vector. 
A

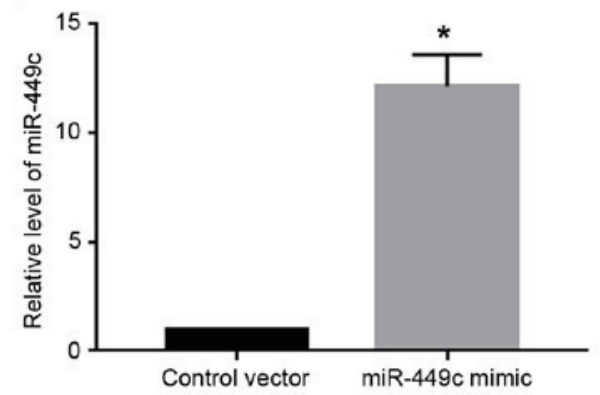

B
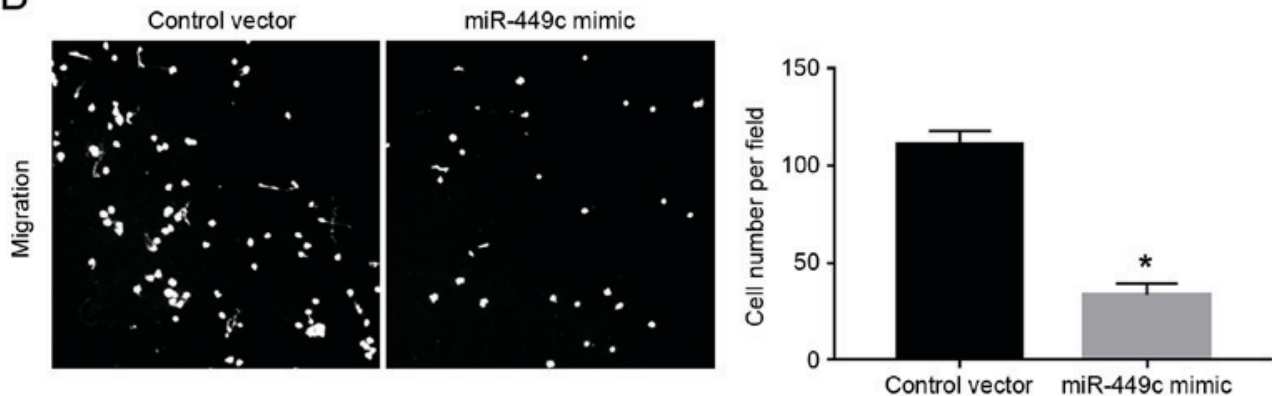

C
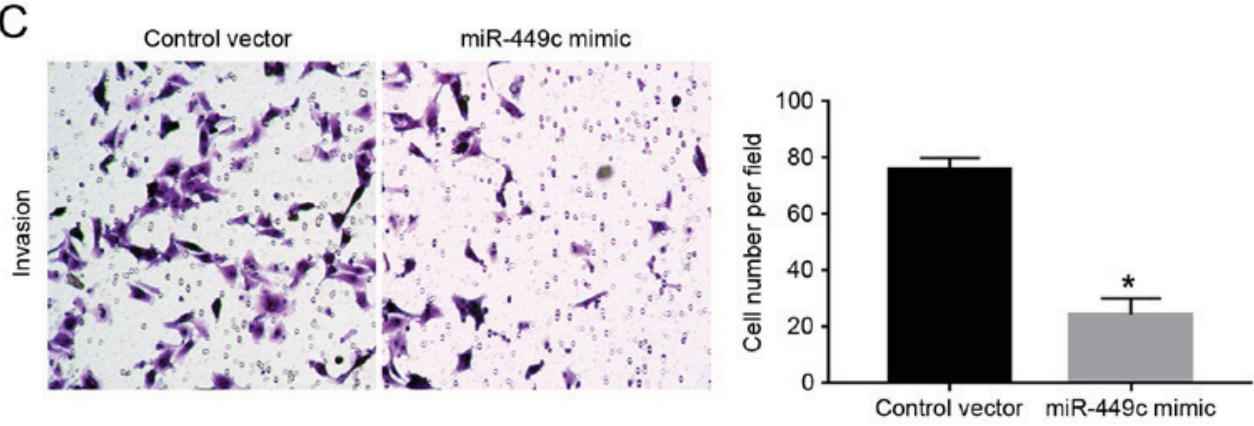

Figure 2. Overexpression of miR-449c inhibits migration and invasion in SGC-7901 cells. (A) SGC-7901 cells were transfected with miR-449c mimic or a control vector and then analyzed for miR-449c expression. ${ }^{*} \mathrm{P}<0.05$. (B) Migration assays revealed that miR-449c overexpression inhibited the migration of SGC-7901 cells. Representative images (magnification, $\mathrm{x} 40$ ) and quantification. ${ }^{*} \mathrm{P}<0.05$. (C) Transwell invasion assays revealed that miR-449c overexpression inhibited the invasion of SGC-7901 cells. Representative images (magnification, $\mathrm{x} 40$ ) and quantification. ${ }^{*} \mathrm{P}<0.05$.

Knockdown of miR-449c inhibits migration and invasion in GC cells. As illustrated in Fig. 1C, the expression levels of miR-449c were the highest in MGC-803 cells. Therefore, MGC-803 cells were transfected with miR-449c inhibitor, which resulted in a significant decrease of miR-449c expression levels in MGC-803 cells $(\mathrm{P}<0.05$; Fig. 3A). Subsequently, miR-449c knockdown significantly enhanced the migration $(\mathrm{P}<0.05$; Fig. 3B) and invasion $(\mathrm{P}<0.05$; Fig. 3C) of MGC-803 cells, compared with cells transfected with control vector.

PFKFB3 is a direct downstream target of miR-449c in GC cells. Following demonstrating the functional role of miR-449c in GC cells, the molecular mechanisms underlying these functions were further explored. Based on predictions using the online bioinformatics database Targetscan (http://www. targetscan.org/vert_71/), the 3'-UTR of the gene PFKFB3 was revealed to contain the binding sequences for miR-449c (Fig. 4A), indicating that PFKFB3 may be a downstream target of miR-449c. To determine whether miR-449c could interact with the PFKFB3 3'-UTR, luciferase assays were performed.
Overexpression of miR-449c in SGC-7901 cells significantly reduced the luciferase activity of wt PFKFB3 3'-UTR, but had no effect on that of mt PFKFB3 3'-UTR ( $\mathrm{P}<0.05$; Fig. 4B). By contrast, miR-449c knockdown in MGC-803 cells significantly increased the luciferase activity of wt PFKFB3 3'-UTR, but had no effect on that of mt PFKFB3 3'-UTR $(\mathrm{P}<0.05$; Fig. 4C). Western blot analysis for the protein expression levels of PFKFB3 in GC cell lines demonstrated that MGC-803 cells, which had the highest miR-449c levels, exhibited the lowest PFKFB3 levels, while SGC-7901 cells, which had the lowest miR-449c levels, exhibited the highest PFKFB3 levels (Fig. 4D). Furthermore, RT-qPCR and western blot analyses were performed to investigate whether miR-449c could regulate the expression of PFKFB3 in GC cells. The results demonstrated that miR-449c overexpression significantly decreased the mRNA $(\mathrm{P}<0.05$; Fig. 5A) and protein $(\mathrm{P}<0.05$; Fig. 5B) expression levels of PFKFB3 in SGC-7901 cells. By contrast, miR-449c knockdown significantly increased the mRNA ( $\mathrm{P}<0.05$; Fig. 5C) and protein $(\mathrm{P}<0.05$; Fig. 5D) expression levels of PFKFB3 in MGC-803 cells. 
A

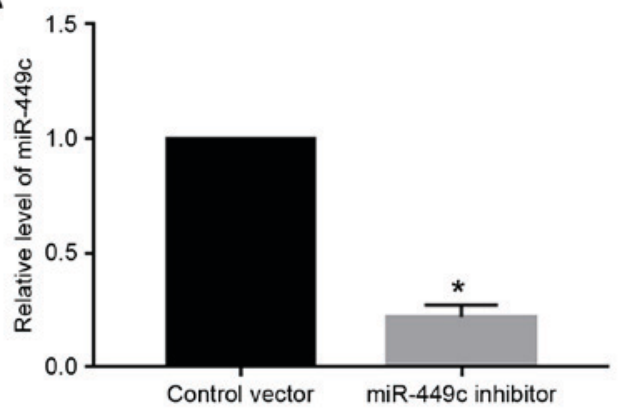

B
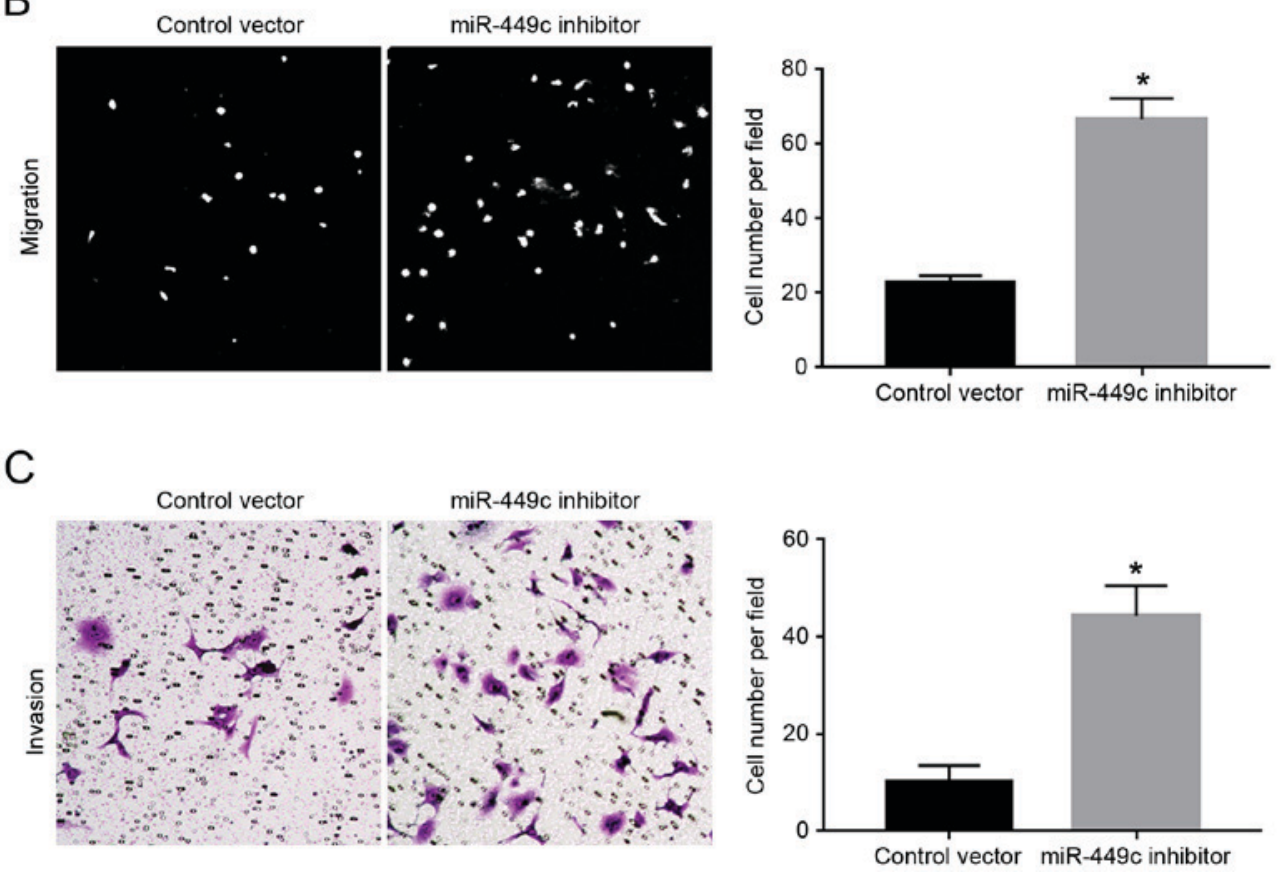

Figure 3. Knockdown of miR-449c promotes migration and invasion in MGC-803 cells. (A) MGC-803 cells were transfected with miR-449c inhibitor or a negative control vector and then analyzed for miR-449c expression. " $\mathrm{P}<0.05$. (B) Migration assays revealed that miR-449c knockdown increased the migration of MGC-803 cells. Representative images magnification, $\mathrm{x} 40$ ) and quantification. ${ }^{*} \mathrm{P}<0.05$. (C) Transwell invasion assays revealed that miR-449c knockdown increased the invasion of MGC-803 cells. Representative images (magnification, $\mathrm{x} 40$ ) and quantification. ${ }^{*} \mathrm{P}<0.05$.

Overexpression of PFKFB3 abrogates the effect of $m i R-449 c$ in $G C$ cells. To further confirm whether PFKFB3 is the mediator of the biological function of miR-449c in GC cells, SGC-7901 cells overexpressing miR-449c were transfected with PFKFB3 expression vector. Transfection of the PFKFB3 vector significantly increased the protein expression levels of PFKFB3 in SGC-7901 cells overexpressing miR-449c $(\mathrm{P}<0.05$; Fig. 6A). Overexpression of PFKFB3 significantly inhibited the migration $(\mathrm{P}<0.05$; Fig. 6B) and invasion $(\mathrm{P}<0.05$; Fig. $6 \mathrm{C})$ of SGC-7901 cells overexpressing miR-449c. The present results suggest that PFKFB3 overexpression abrogated the inhibitory effects of miR-449c on the migration and invasion of SGC-7901 cells.

\section{Discussion}

miRNAs have been demonstrated as important regulators of the development and progression of human cancer types (4). miRNAs have been reported to regulate the growth, metastasis, stem cell biology and drug resistance of cancer cells $(9,14,15)$.
Among numerous miRNAs, miR-449c was identified as a novel cancer-related microRNA, and it was reported to inhibit the proliferation and invasion ability of non-small cell lung cancer cells (11). In liver cancer, miR-449c was reported to inhibit the epithelial-mesenchymal transition and metastasis of liver cancer cells (12). However, the expression and function of miR-449c in GC remained unknown. The present study demonstrated that miR-449c is downregulated in GC tissues and cells compared with normal tissues and cells. Functionally, both gain and loss-of function assays demonstrated that miR-449c inhibited the migration and invasion of GC cells. The present data indicate that miR-449c may exert tumor suppressive roles in GC by inhibiting the migration and invasion of GC cells.

6-phosphofructo-2-kinase (PFKFB3) has been reported to be a versatile protein in human cancers $(16,17)$. It has been demonstrated to regulate the glycolysis, oxidative stress, proliferation, apoptosis and metastasis of cancer cells (16). However, the underlying mechanisms for the elevated expression of PFKFB3 in GC remain unknown. A previous study 
A
hsa-miR-449c

wt PFKFB3 3'-UTR

mt PFKFB3 3'-UTR

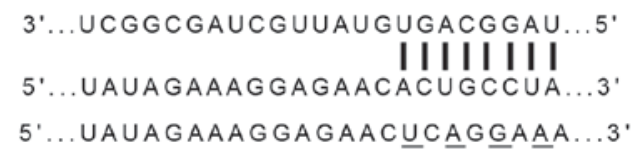

B

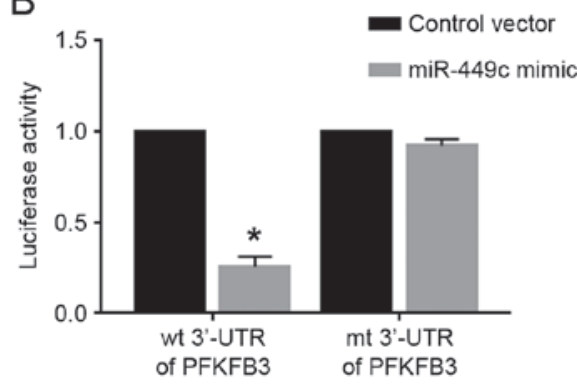

C

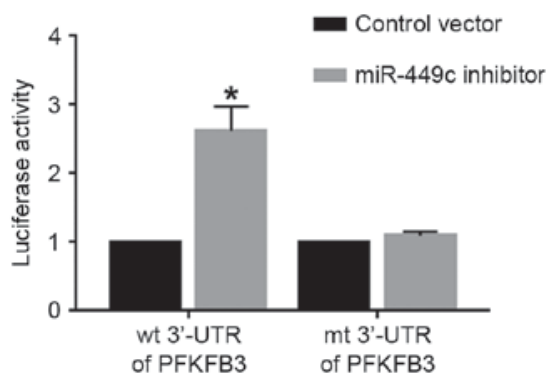

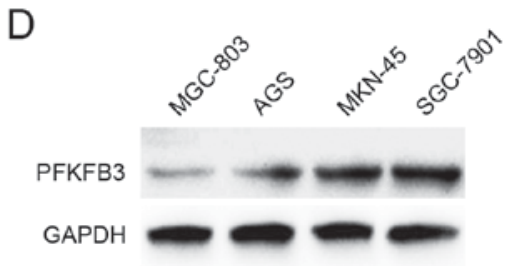

Figure 4. miR-449c interacts with the PFKFB3 3'-UTR in GC cells. (A) Schematic of the predicted binding sequences of miR-449c in the wt 3'UTR of PFKFB3. Underlined parts denote the mutant sites designed for the generation of the mt PFKFB3 3'UTR sequence. (B) Overexpression of miR-449c in SGC-7901 cells decreased the luciferase activity of wt PFKFB3 3'-UTR, while it had no effect on that of mtPFKFB3 3'-UTR. "P $<0.05$ compared with control. (C) Inhibition of miR-449c in MGC-803 cells increased the luciferase activity of wt PFKFB3 3'-UTR, while it had no effect on that of mt PFKFB3 3'-UTR. "P<0.05 compared with control. (D) The protein expression levels of PFKFB3 in GC cell lines were examined by western blotting. PFKFB3, 6-phosphofructo-2-kinase; UTR, untranslated region; GC, gastric cancer; wt, wild type; mt, mutant.

A

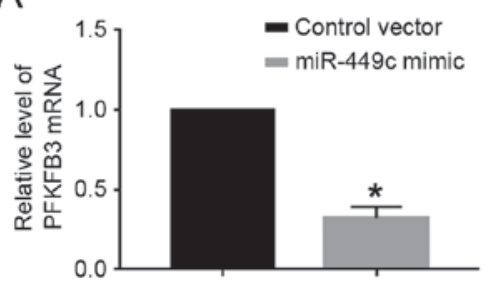

C

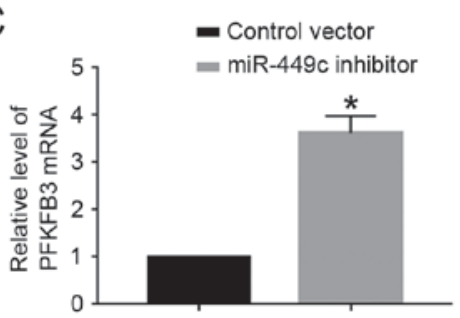

B
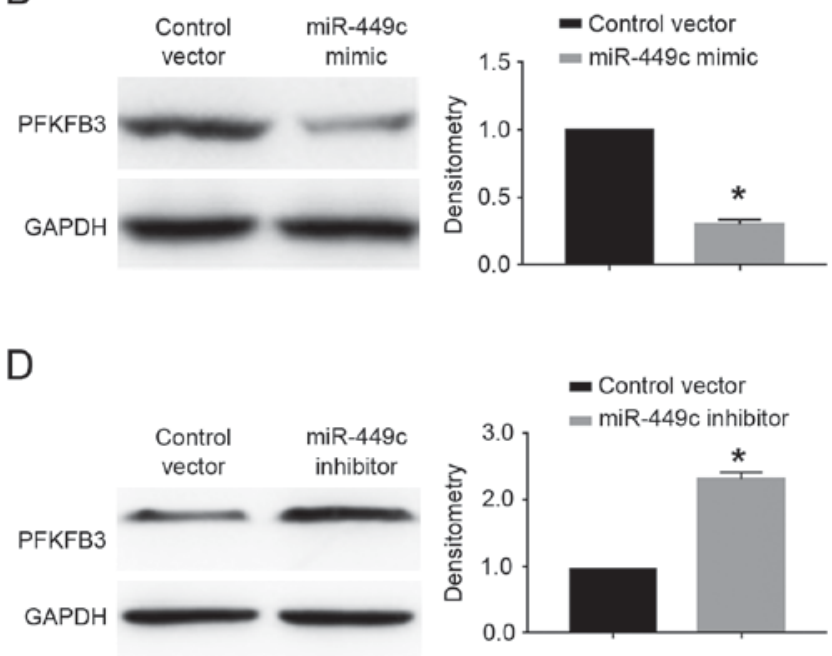

Figure 5. miR-449c regulates the expression of PFKFB3 in GC cells. (A) Overexpression of miR-449c in SGC-7901 cells decreased the PFKFB3 mRNA levels in SGC-7901 cells. "P<0.05. (B) Overexpression of miR-449c in SGC-7901 cells decreased the PFKFB3 protein levels in SGC-7901 cells. "P<0.05. (C) Knockdown of miR-449c in MGC-803 cells increased the PFKFB3 mRNA levels in MGC-803 cells. "P<0.05. (D) Knockdown of miR-449c in MGC-803 cells increased the PFKFB3 protein levels in MGC-803 cells. ${ }^{*} \mathrm{P}<0.05$.

has reported that PFKFB3 is under the regulation of miR-26b in osteosarcoma cells. In the present study, PFKFB3 was demonstrated to be the downstream target of miR-449c in GC cells. PFKFB3 overexpression abrogated the inhibitory effects of miR-449c on the migration and invasion of GC cells. These data suggest that PFKFB3 may have mediated the inhibitory effect of miR-449c on the migration and invasion of GC cells. Notably, previous studies of non-small cell lung cancer and liver cancer cells have reported that c-Myc and SOX4 were the downstream targets of miR-449c, respectively, suggesting that 
A

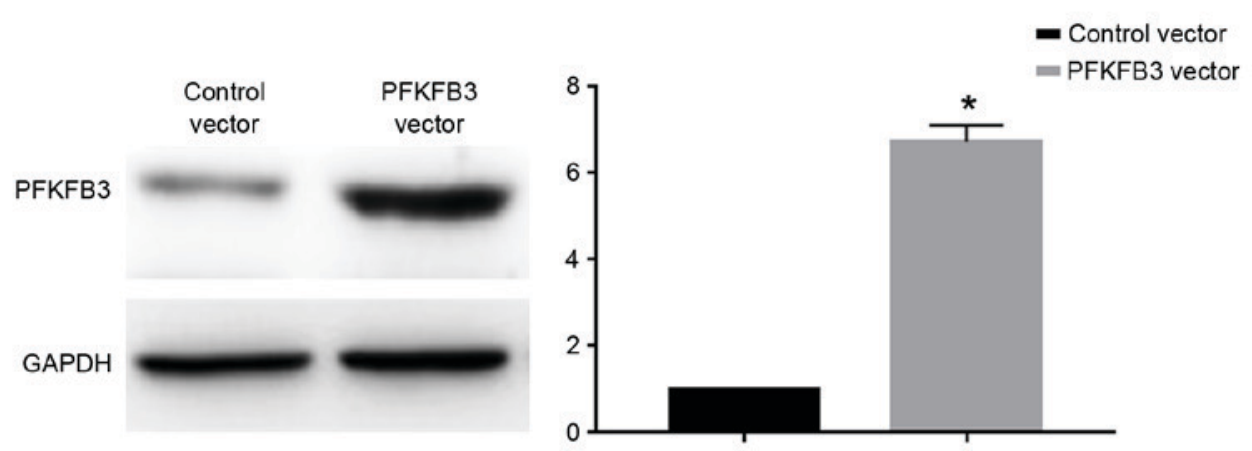

B
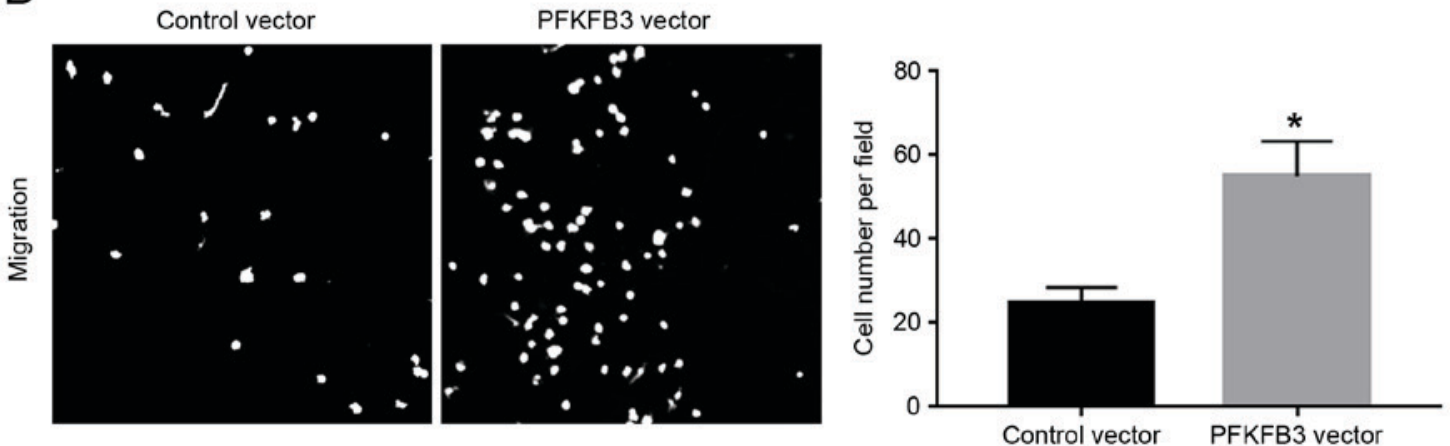

C
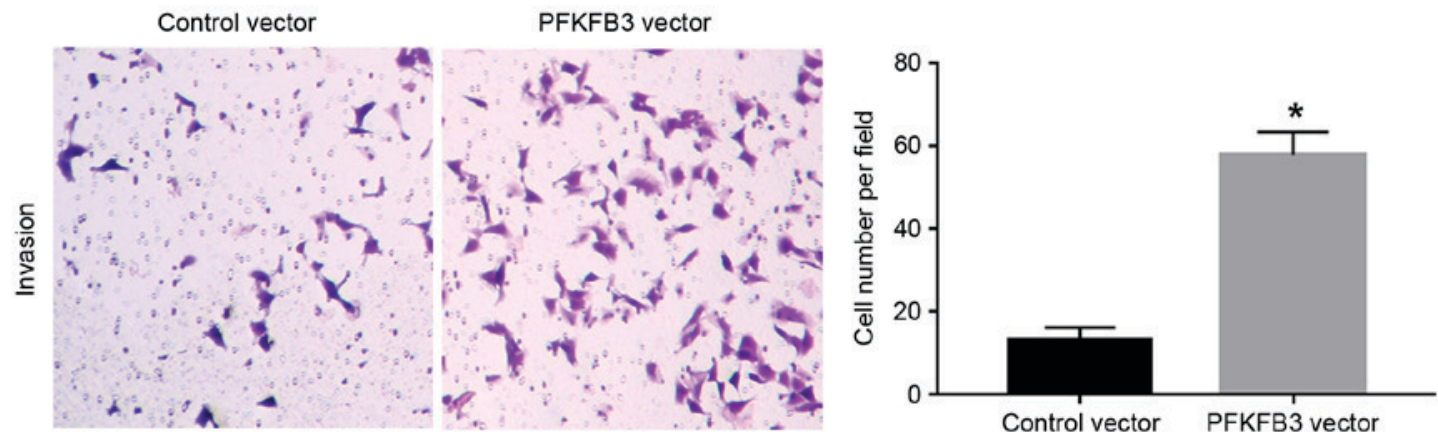

Figure 6. Overexpression of PFKFB3 abrogates the inhibitory effect of miR-449c on the migration and invasion of SGC-7901 cells. (A) The PFKFB3 expression vector significantly increased the PFKFB3 protein expression levels in SGC-7901 cells transfected with miR-449c mimic. ${ }^{*} \mathrm{P}<0.05$. (B) PFKFB3 overexpression significantly increased the migration of SGC-7901 cells transfected with miR-449c mimic. Representative images (magnification, $\mathrm{x} 40$ ) and quantification. " $\mathrm{P}<0.05$. (C) PFKFB3 overexpression significantly increased the invasion of SGC-7901 cells transfected with miR-449c mimic. Representative images (magnification, $\mathrm{x} 40$ ) and quantification. ${ }^{*} \mathrm{P}<0.05$.

miR-449c may have different downstream targets in different types of human cancers.

In summary, the present study demonstrated that miR-449c was downregulated in GC tissues and cells. Decreased expression of miR-449c was associated with poor prognosis in GC patients. Overexpression of miR-449c inhibited migration and invasion in SGC-7901 cells, while knockdown of miR-449c promoted these biological behaviors in MGC-803 cells. Finally, PFKFB3 was revealed as a novel downstream target and functional mediator of miR-449c in GC cells.

\section{Acknowledgements}

Not applicable.

\section{Funding}

No funding was received.

\section{Availability of data and materials}

The datasets used and/or analyzed during the current study are available from the corresponding author on reasonable request.

\section{Authors' contributions}

$\mathrm{XC}$ performed the experiments in the present study, AW performed the data analysis, and $\mathrm{XC}$ and $\mathrm{XY}$ designed this study and wrote the manuscript. 


\section{Ethics approval and consent to participate}

Ethical approval was obtained from the Institutional Research Ethics Committee of the Second Affiliated Hospital of Xinjiang Medical University. Informed consent to participate in the study was obtained from participants.

\section{Consent for publication}

All participants agreed to the publication of this study.

\section{Competing interests}

The authors declare that they have no competing interests.

\section{References}

1. Rugge M, Fassan M and Graham DY: Epidemiology of gastric cancer. In: Gastric Cancer Springer, pp23-34, 2015.

2. Chang WJ, Du Y, Zhao X, Ma LY and Cao GW: Inflammation-related factors predicting prognosis of gastric cancer. World J Gastroenterol 20: 4586-4596, 2014.

3. Ha M and Kim VN: Regulation of microRNA biogenesis. Nat Rev Mol Cell Biol 15: 509-524, 2014.

4. Lin S and Gregory RI: MicroRNA biogenesis pathways in cancer. Nat Rev Cancer 15: 321-333, 2015.

5. Ameres SL and Zamore PD: Diversifying microRNA sequence and function. Nat Rev Mol Cell Biol 14: 475-488, 2013.

6. Dong H, Lei J, Ding L, Wen Y, Ju H and Zhang X: MicroRNA: Function, detection, and bioanalysis. Chem Rev 113: 6207-6233, 2013.
7. Acunzo M, Romano G, Wernicke D and Croce CM: MicroRNA and cancer-a brief overview. Adv Biol Regul 57: 1-9, 2015.

8. Wen D, Danquah M, Chaudhary AK and Mahato RI: Small molecules targeting microRNA for cancer therapy: Promises and obstacles. J Control Release 219: 237-247, 2015.

9. Hayes J, Peruzzi PP and Lawler S: MicroRNAs in cancer: Biomarkers, functions and therapy. Trends Mol Med 20: 460-469, 2014.

10. Zhao X, Dou W, He L, Liang S, Tie J, Liu C, Li T, Lu Y, Mo P, Shi Y, et al: MicroRNA-7 functions as an anti-metastatic microRNA in gastric cancer by targeting insulin-like growth factor-1 receptor. Oncogene 32: 1363-1372, 2013.

11. Miao LJ, Huang SF, Sun ZT, Gao ZY, Zhang RX, Liu Y and Wang J: MiR-449c targets c-Myc and inhibits NSCLC cell progression. FEBS Lett 587: 1359-1365, 2013.

12. Sandbothe M, Buurman R, Reich N, Greiwe L, Vajen B, Gürlevik E, Schäffer V, Eilers M, Kühnel F, Vaquero A, et al: The microRNA-449 family inhibits TGF- $\beta$-mediated liver cancer cell migration by targeting SOX4. J Hepatol 66: 1012-1021, 2017.

13. Livak KJ and Schmittgen TD: Analysis of relative gene expression data using real-time quantitative PCR and the 2(-Delta Delta C(T)) method. Methods 25: 402-408, 2001.

14. Adams BD, Kasinski AL and Slack FJ: Aberrant regulation and function of microRNAs in cancer. Curr Biol 24: R762-R776, 2014

15. Farazi TA, Hoell JI, Morozov P and Tuschl T: MicroRNAs in human cancer. In: MicroRNA Cancer Regulation Springer, pp1-20, 2012.

16. Clem BF, O'Neal J, Tapolsky G, Clem AL, Imbert-Fernandez Y, Kerr DA II, Klarer AC, Redman R, Miller DM, Trent JO, et al: Targeting 6-phosphofructo-2-kinase (PFKFB3) as a therapeutic strategy against cancer. Mol Cancer Ther 12: 1461-1470, 2013.

17. Imbert-Fernandez Y, Clem A, Clem B, Tapolsky G, Telang S and Chesney J: Suppression of 6-Phosphofructo-2-Kinase (PFKFB3) for the treatment of breast cancer. AACR 76, 2016. 